\title{
FUNCIONES PARA LA ESTIMACION DE LA BIOMASA TOTAL Y DE COMPONENTES DEL QUILLAY (Quillaja saponaria Mol)
}

\author{
José Antonio Prado D.* \\ Sergio Aguirre A.
}

\begin{abstract}
RESUMEN
El Quillay es una de las especies más interesantes que crecen en la zona semiárida de Chile. Sin embargo, los actuales métodos de explotación de la especie se caracterizan por un bajo aprovechamiento de la biomasa del árbol. Para lograr un mejor manejo y uso de esta especie, es necesario conocer su potencial productividad, es decir, poder estimar su rendimiento mediante funciones de peso.

El objetivo de este trabajo es determinar las funciones de biomasa que mejor estimen el peso de los árboles, para lo cual se muestrearon y pesaron completamente 50 árboles de Quillay, seleccionados de tres zonas de crecimiento de la especie. Para registrar el peso de estos árboles muestra, se separaron y pesaron en forma independiente los principales componentes que conforman el árbol, es decir, fuste, corteza, ramas y ramillas.

Para determinar las mejores funciones de biomasa, se probaron modelos matemáticos lineales y alométricos, procesándolos mediante el método de regresión paso a paso. Las funciones calculadas fueron analizadas y comparadas usando varios indicadores estadísticos.

Las mejores funciones obtenidas fueron las basadas en modelos lineales múltiples, que entregaron resultados satisfactorios en la estimación del peso total y de corteza total del árbol.
\end{abstract}

\section{ABSTRACT}

Quillaja is one of the most important species growing in the semiarid region of Chile. However, full biomass of the tree is not being utilized due to deficient explotation methods. Biomass equations to estimate the weight of total stem bark, stem wood, branches and total tree were developed. Data was obtained from in three sites of the growing area of the species and fifty trees were completely wighted. Individual tree components and total tree weights were regressed using linear and allometric models. The equations thus calculated were compared through statistic analysis. The best results were obtained with multiple linear models, giving better estimates for total tree and stem bark weights.

* Ingenieros Forestales, U. de Chile. División de Silvicultura, Instituto Forestal. Huérfanos 554, Piso 4. Santiago - Chile. 


\section{INTRODUCCION}

El Quillay (Quillaja saponaria Mol), una de las especies nativas de la zona semiárida chilena que tiene importancia económica, se ha explotado comercialmente para aprovechar su corteza, de la que se extrae una saponina de múltiples usos.

La explotación se ha venido realizando desde principios del siglo pasado. Ya entre 1844 y 1864 , se explotaban en promedio unas 300 toneladas de corteza por año, volumen que a principios de este siglo llegó a unas 2.100 toneladas por año (Neuenschwander, 1965).

Esta extracción, realizada muchas veces sin técnicas ni control adecuados, unida al sobrepastoreo, que limita fuertemente su regeneración, han ido produciendo una disminución progresiva de la especie, especialmente en la parte norte de su área de distribución.

Considerando que la corteza comercial de un árbol de tamaño medio puede alcanzar 18 a $20 \mathrm{~kg}$ en peso seco, la obtención de 2.100 toneladas al año significa la corta de unos $110 \mathrm{mil}$ árboles. Los bajos rendimientos en corteza comercial se deben a los sistemas de explotación empleados y a las exigencias del mercado, que sólo acepta corteza en pedazos grandes. Toda la corteza de las ramas que tienen menos de $15 \mathrm{~cm}$ de diámetro es normalmente desechada, de modo que se desperdicia una gran cantidad de materia prima adecuada para la obtención de saponina.

Las ramas y hojas también contienen saponina y otra serie de productos, aunque en menor cantidad que la corteza (Vidal, 1945), pero hay que considerar que la mayor parte de la biomasa del árbol se encuentra en estos componentes.

El desarrollo de sistemas de obtención de saponinas que permitan utilizar más efícientemente la materia prima, con miras a exportar un producto más elaborado y no sencillamente la corteza, es un paso fundamental no sólo para la protección de la especie, sino también para promover su plantación, que con los sistemas actuales de utilización no es una inversión económicamente atractiva.

La falta de una herramienta adecuada que permita estimar la productividad potencial de la especie es una de las razones que hacen difícil este desarrollo.

El presente trabajo pretende hacer un aporte en este sentido, al entregar funciones que permiten estimar el peso total y de los distintos componentes del árbol de Quillay. Conociendo los contenidos de saponina de cada componente, se pueden estimar los rendimientos que una utilización racional permitiría general, así como la cantidad de leña o carbón que es posible obtener de la madera más gruesa, si no se la emplea para la obtención de saponinas.

\section{MATERIAL Y METODO}

a) Muestreo: Para la determinación de las funciones de biomasa se empleó una muestra de 50 árboles, obtenidos de tres áreas donde se realizaban explotaciones comerciales de Quillay, ubicados en las cercanias de Sagrada Familia (VII Región), Santa Cruz (VI Región) y Casablanca (V Región).

Con el fin de conocer las características que presentan las formaciones de Quillay, en cuanto a su desarrollo y a su distribución en clases diamétricas, se analizaron los datos de ocho inventarios realizados en las áreas donde se realizan explotaciones. Los árboles se agruparon en 5 clases diamétricas, determinando una tabla promedio, que se presenta en el Cuadro No 1 .

Para asegurar una buena distribución de los árboles que se iban a muestrear en las distintas clases de diámetro, 25 árboles fueron distribuidos en forma homogénea en las 5 clases. Los 25 árboles restantes fueron distribuidos en forma aleatoria, pero con una probabilidad proporcional al número de árboles por clase (F) multiplicado por el diámetro (DAP), lo que tenía por objetivo dar una mayor probabilidad de asignación a aquellas clases de mayor frecuencia y de diámetros mayores. 


\section{CUADRO 1}

\section{TABLA DE RODAL Y NUMERO DE ARBOLES ASIGNADOS POR CLASE DIAMETRICA}

\begin{tabular}{ccc}
\hline $\begin{array}{c}\text { Clases } \\
\text { de } \\
\text { DAP }\end{array}$ & $\begin{array}{c}\text { No de } \\
\text { árboles/ha } \\
(\mathbf{1})\end{array}$ & $\begin{array}{c}\text { No de } \\
\text { árboles } \\
\text { muestra }\end{array}$ \\
\hline $1-20$ & 38 & 11 \\
$21-40$ & 36 & 12 \\
$41 \cdot 60$ & 15 & 8 \\
$61-80$ & 9 & 12 \\
$81-100$ & 1 & 7 \\
Total & & $\mathbf{5 0}$ \\
\hline
\end{tabular}

NOTA (1): Vaiores obtenidos de 8 inventarios.

b) Obtención de peso verde y peso seco: En los lugares de muestreo se identificó un número variable de árboles de cada clase diamétrica y de entre éstos se eligieron, al azar, los que serían muestreados. En aquellos casos en que un árbol presentaba dos o más fustes que salían desde el nivel del suelo, cada uno de ellos fue considerado en forma separada.

Antes de cortarlos, se midieron las siguientes variables: Altura total (HT); Diámetro basal (DB); Diámetro a la altura del pecho (DAP); Número de ramas principales (diámetro $>$ a $15 \mathrm{~cm}$ ) (NR); Peso de corteza total (PCT) y Peso de corteza comercial (PCC). Esta última se obtiene extrayendo todo el ritidoma, es decir, todos los tejidos muertos exteriores. Estos pesos se registraron después de cosechado cada árbol por las cuadrillas que realizaban explotaciones comerciales en las áreas de muestreo.

Los árboles fueron descortezados en pie, hasta un diámetro menor de fuste y ramas de alrededor de $15 \mathrm{~cm}$, y luego se procede a su corta.

Una vez cortado, se le midió el largo total (LT) y el largo del fuste comercial (hasta $15 \mathrm{~cm}$ de diámetro) (LC).

Para la obtención del peso verde, el árbol fue dividido en los siguientes componentes:

a) Fuste : Comprende el tronco principal y todas sus ramificaciones hasta un diámetro de $15 \mathrm{~cm}$, sin corteza.

b) Ramas : Ramas principales y secundarias con diámetros máximos inferiores a 15 cm y mínimos de $3 \mathrm{~cm}$, con corteza.

c) Ramillas : Ramas secundarias con diámetros inferiores a $3 \mathrm{~cm}$. Este componente incluye hojas y frutos.

c) Corteza : Como ya se mencionó, se divide en corteza total, que corresponde a la corteza presente en el fuste y las ramas hasta un diámetro de $15 \mathrm{~cm}, \mathrm{y}$ corteza comercial, que es la parte útil de la corteza total.

Para obtener una relación que permitiera estimar el peso seco se tomaron muestras de cada componente. Del fuste y de las ramas se obtuvieron 4 a 6 discos, de $5 \mathrm{~cm}$ de espesor, los que fueron inmediatamente pasados con una precisión de $0,1 \mathrm{~g}$. Asimismo se seleccionaron al azar 3 ramillas completas, de las cuales se obtuvieron muestras para determinar la relación peso 
verde/peso seco, y se estimó la proporción del peso total de la ramilla correspondiente a las hojas. Muestras de corteza total y comercial también fueron pasadas en estado verde.

Cada componente fue pesado en su totalidad, empleando una romana y una balanza de reloj, con una precisión de $100 \mathrm{~g}$.

Todas estas muestras se secaron en hornos a $100^{\circ} \mathrm{C}$, hasta obtener peso constante. El porcentaje de corteza en las ramas fue determinado empleando los discos de la muestra.

c) Análisis: Para determinar las funciones que permitieran estimar el peso total y de los distintos componentes de la especie en estudio, se probaron diversos modelos, empleando las variables independientes tal como fueron medidas, combinadas o transformadas.

Se probaron modelos alométricos del tipo:

$$
\mathrm{Y}=\mathrm{b}_{\mathrm{O}} \mathrm{X}^{\mathrm{b}_{1} \mathrm{C}}
$$

que fueron ajustados como modelos lineales, realizando previamente una transformación logarítmica, y modelos lineales de la forma:

$$
\mathrm{Y}=\mathrm{b}_{\mathrm{o}}+\mathrm{b}_{1} \mathrm{X}_{1}+\mathrm{b}_{2} \mathrm{X}_{2}+\ldots \ldots+\mathrm{b}_{\mathrm{n}} \mathrm{X}_{\mathrm{n}}
$$

La selección de las variables que se incluirían en los diferentes modelos se hizo empleando el método de regresión paso a paso (Step-Wise).

Para selecionar las mejores funciones se compararon los valores del Coeficiente de Determinación $\left(\mathrm{R}^{2}\right)$, los Errores Cuadráticos Medios y la proporción de observaciones con errores menores al 10, 15 y $20 \%$ o. También se consideró la presencia de sesgos. Entre todos estos indicadores se dio mayor importancia al Error Cuadrático Medio, ya que incluye la totalidad de los errores, aleatorios y sistemáticos, y por lo tanto se constituye en el mejor indicador de la exactitud de la función.

\section{RESULTADOS}

Las funciones seleccionadas para estimar el peso total y de los componentes del árbol se indican en el siguiente cuadro 2.

\section{CUADRO 2: \\ FUNCIONES DE BIOMASA PARA ESTIMAR PESO VERDE TOTAL Y DE COMPONENTES DEL QUILLAY. (PESO VERDE EN KG)}

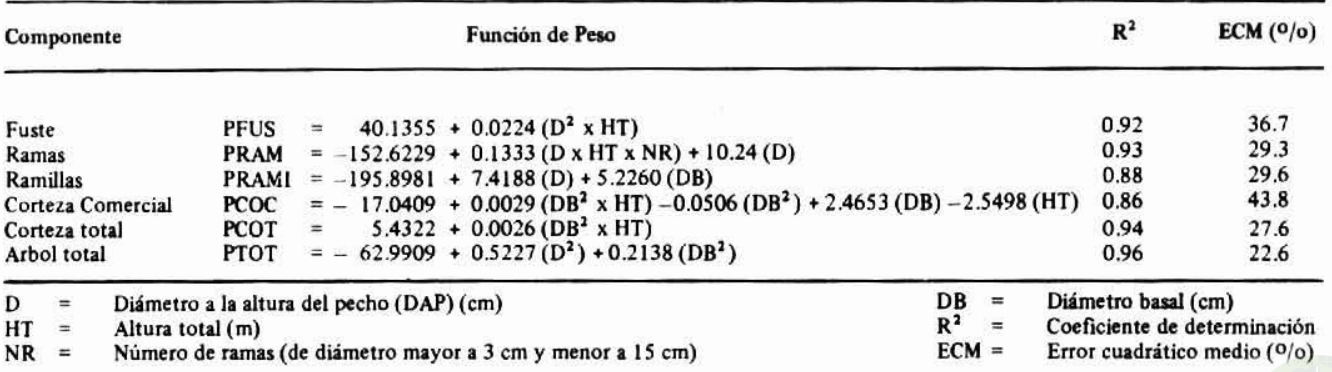


Para obtener el peso seco a partir de estas funciones indicadas en el Cuadro 2, los resultados de peso verde deben ser modificados de acuerdo con los factores de conversión peso seco/ peso verde, que se indican en el Cuadro 3.

\section{CUADRO 3: \\ FACTORES DE CONVERSION DE PESO VERDE \\ A PESO SECO}

\begin{tabular}{|l|c|}
\hline Componente & Factor \\
\hline Madera fuste & 0,51 \\
Ramas & 0,49 \\
Ramillas & 0,51 \\
Corteza total & 0,65 \\
Corteza comercial & 0,59 \\
\hline
\end{tabular}

\section{DISCUSION}

A diferencia de la mayoría de los estudios de biomasa para otras especies, los modelos lineales proporcionaron en este caso mejores estimaciones de peso que los modelos logarítmicos. A pesar de las correcciones por sesgo realizadas, (Baskerville, 1972), las funciones obtenidas con modelos lineales presentaron sesgos menores que los logarítmicos, ocurriendo algo similar con el Error Cuadrático Medio (ECM).

Por otra parte, las funciones logarítmicas mostraron una alta proporción de observaciones con errores menores al 5, 10 y $20 \%$ o. Los Coeficientes de Determinación $\left(\mathrm{R}^{2}\right)$ son bastante similares, sólo levemente superiores en el caso de las funciones logarítmicas. Se probaron también funciones ponderadas empleando el factor $\left(1 / \mathrm{DB}^{2} \mathrm{H}\right)$, pero tampoco se obtuvo una mejoría notable en las estimaciones.

La mejor estimación se obtiene para el peso total del árbol, mientras que la función para la corteza comercial presenta la mas baja correlación y los más altos errores; esto se explica en gran medida por el hecho de que la obtención de este componente depende de factores ajenos al tamaño de los árboles.

Debido al empleo de distintos modelos para calcular el peso de los componentes del árbol, las funciones seleccionadas no son aditivas (Kozak, 1970), es decir, la suma de las estimaciones del peso de los componentes no es igual a la estimación del peso total. Para árboles pequeños, la suma del peso de los componentes entrega valores inferiores a los obtenidos mediante la función de peso total; lo contrario ocurre en los árboles grandes.

De acuerdo con Williams (1982), la aditividad en ecuaciones de biomasa es un factor importante que se debe considerar. A fin de cumplir con esta condición, se elaboró un modelo simple con las variables que más se repitieron en las funciones seleccionadas, las que resultaron $\operatorname{ser}\left(D^{2} H T\right)$ y $\left(D^{2}\right)$. Estas funciones, que se indican en el Cuadro 4 , presentan aditividad, pero con una pérdida en términos del error, especialmente en el caso de ramas y ramillas. 


\section{CUADRO 4: \\ FUNCIONES DE BIOMASA PARA ESTIMAR EL PESO VERDE DEL QUILLAY, A BASE DE UN MODELO LINEAL MULTIPLE DEL TIPO

$$
\mathrm{Y}=\mathrm{b}_{\mathrm{O}}+\mathrm{b}_{1}\left(\mathrm{D}^{2} \mathrm{H}\right)+\mathrm{b}_{2}\left(\mathrm{D}^{2}\right)
$$

\begin{tabular}{llccc}
\hline Componente & & Función de Peso $(\mathbf{k g})$ & $\mathbf{R}^{2}$ & ECM $(\mathbf{0} / \mathbf{o})$ \\
\hline Fuste & PFUS $=$ & $16.3301+0.0185\left(\mathrm{D}^{2} \times \mathrm{HT}\right)+0.0636\left(\mathrm{D}^{2}\right)$ & 0.92 & 36.5 \\
Ramas & PRAM $=$ & $-53.7017-0.0158\left(\mathrm{D}^{2} \times \mathrm{HT}\right)+0.4568\left(\mathrm{D}^{2}\right)$ & 0.88 & 37.2 \\
Ramillas & PRAMI $=$ & $52.0020-0.0063\left(\mathrm{D}^{2} \times \mathrm{HT}\right)+0.2266\left(\mathrm{D}^{2}\right)$ & 0.86 & 32.8 \\
Corteza total & PCOT $=$ & $28.0926+0.0045\left(\mathrm{D}^{2} \times \mathrm{HT}\right)-0.0255\left(\mathrm{D}^{2}\right)$ & 0.85 & 44.3 \\
Arbol total & PTOT $=$ & $40.0464-0.0011\left(\mathrm{D}^{2} \times \mathrm{HT}\right)+0.7737\left(\mathrm{D}^{2}\right)$ & 0.95 & 25.5 \\
\hline
\end{tabular}

$\mathrm{D}=$ Diámetro a la altura del pecho (DAP) $(\mathrm{cm})$

$\mathrm{HT}=$ Altura total $(\mathrm{m})$

$\mathrm{R}^{2}=$ Coeficiente de determinación

$\mathrm{ECM}=\quad$ Error cuadrático medio.

\section{CONCLUSIONES}

Las funciones seleccionadas permiten estimar razonablemente el peso total y de componentes del árbol de Quillay, dentro de los rangos de medida de las variables consideradas en este estudio. A su vez, mediante la incorporación de más datos a la muestra original, se espera en el futuro mejorar la precisión de estimación de estas funciones.

En general, la metodología aplicada en este trabajo entrega resultados satisfactorios y puede ser utilizada para desarrollar funciones de biomasa para otras especies.

Mediante el uso progresivo de estas funciones en la planificación de las explotaciones de Quillay, se espera disminuir en el futuro el deterioro a que está siendo sometida esta especie, ya que al realizarse un aprovechamiento integral del árbol, se podrá racionalizar su corta, lo que también favorecerá su regeneración y manejo.

\section{Agradecimientos}

Este trabajo de investigación forma parte de un proyecto apoyado por el Centro Internacional de Investigaciones para el Desarrollo de Canadá.

Los autores agradecen a todas aquellas personas que participaron en la toma de datos y colaboraron en su análisis. 


\section{REFERENCIAS BIBLIOGRAFICAS}

1. BASKERVILLE, G.L. 1972. Use of logarithmic regression in the estimation of plant biomass. Can. J. For. Res. 2:49-53.

2. CROW, T.R. and LAIDLY, P.R. 1980. Alternative models for estimating woody plant biomass. Can. J. For. Res. 10:367-370.

3. KOZAK, A. 1970. Methods for ensuring additivity of biomass components by regression analysis. For. Choron. 46(5):402-404.

4. NEUENSCHWANDER A., A. 1965. Contribución al estudio anatómico de la corteza de quillay Quillaja saponaria Mol.) y recomendaciones sobre su explotación. Tesis, Universidad de Chile, Fac. de Agronomía. Santiago. $121 \mathrm{p}$.

5. SCHLAEGEL, B.E. 1982. Boxelder (Acernegundo L.) biomass component regression analysis for the Mississippi Delta. For. Sci. 28(2):355-358.

6. VIDAL A., M. 1945. Estudio químico y obtención de saponias de quillay. Tesis, Universidad de Chile, Fac. de Química y Farmacia. Santiago. 30 p.

7. WIANT, H.V. and HARNER, E.J. 1979. Percent bias and standard error in logarithmic regression. For. Sci. 25(1):167-168.

8. WILLIAMS, J.G., Jr. 1982. Modeling problems in predicting total tree and tree component biomass. In: Proc. of the 1982 Southern Forest Biomass Working Group Workshop. Alexandria, Louisiana, USA. pp. 11-115. 